\title{
A New Definition of Multi-Infeed Short Circuit Ratio Considering Control Modes of DC System
}

\author{
Kang Zhao ${ }^{1, a}$, Dong Yang ${ }^{2, b}$ and Yutian Liü, c \\ ${ }^{1}$ Key Laboratory of Power System Intelligent Dispatch and Control of Ministry of Education \\ (Shandong University), Jinan 250061, China; ${ }^{2}$ State Grid Shandong Electric Power Research \\ Institute, Jinan 250002, China \\ azhaokang1990@gmail.com, byangdong_epri@163.com, cliuyt@sdu.edu.cn
}

Keywords: AC/DC hybrid power system; Multi-infeed interaction factor; Multi-infeed short circuit ratio; Control mode of DC system

\begin{abstract}
The mathematical relationship between reactive power consumed by DC system and AC voltage on inverter bus is derived under different control modes, respectively. Based on these relationships, a new definition of multi-infeed short circuit ratio (MISCR) is formulated from the power-flow model of PQ decoupled method. The specific influence of different control modes on voltage stability is analyzed. Considering the relationship between MISCR and voltage stability of AC/DC hybrid system, the characteristics of values of new MISCR are derived. Case study of an actual regional power grid validates these characteristics.
\end{abstract}

\section{Introduction}

With energy resource dispersing far away from the load centers, HVDC technology has been greatly developed in China over the past decades because of its advantage in long distance and bulk power transmission ${ }^{[1]}$. In the East China Power Grid and the South China Power Grid, the multi-infeed configurations of HVDC have been developed. The interaction between AC and DC systems is very complicated, which threatens the stability and safety of the whole power system ${ }^{[2,3]}$.

An index known as the multi-infeed interaction factor (MIIF) was proposed in [4], which can assess the degree of voltage interaction between inverters. Based on MIIF, reference [5] gives a definition of multi-infeed short circuit ratio (MISCR). MISCR can give a quantitative assessment of the degree of interaction between AC and DC systems. The MIIF proposed in [4], however, is derived from empirical information. Thus, the MISCR defined in [5] cannot be facilitated in rigorous analysis of AC/DC hybrid system. Reference [6,7] propose another definition of MISCR based on the impedance ratio of related nodes other than MIIF. This definition is analytically derived and able to reflect the interaction precisely. Thus, it is widely used in the study about multi-infeed HVDC configuration $^{[8-10]}$. But this definition is a static index based on the impedance matrix. It doesn't reflect the dynamic characteristics of power system elements, which would obviously influence the operation of the AC/DC hybrid system.

In this paper, a new definition of MISCR considering the control modes of DC systems is proposed. Firstly, the Q-V relationship between reactive power consumed by DC system and AC voltage on inverter bus is derived. Then, the power-flow model of PQ decoupled method is used as the mathematical framework to derive the new MISCR based on the Q-V relationship. After the voltage stability under different control mode is analyzed, the characteristics of values of new MISCR are analyzed. Finally, this analysis is validated by the calculation results of an actual power system.

\section{Analysis of Different Control Modes}

In general, there are four control modes for the converters at both ends of the DC transmission line: Constant Current Mode (CC-Mode); Constant Power Mode (CP-Mode); Constant Voltage Mode (CV-Mode); Constant Extinction Angel Mode (CEA-Mode). The previous two control modes (CC-mode and CP-mode) are usually used for rectifier, while the other two modes (CV-mode and 
CEA-mode) are usually used for inverter. Thus, there are four control modes in all for the whole DC system: CC-CV mode, CP-CV mode, CC-CEA mode and CP-CEA mode.

DC systems consumed a great amount of reactive power. A little disturbance of DC system may cause a relatively large voltage change on the converter bus of AC system. Conversely, the converter bus voltage affects the normal operation of DC system greatly. An excessive voltage drop on the converter bus may cause commutation failure even the blocking of DC system. Thus, reactive power characteristics reflects the interaction between AC/DC systems to a large extent ${ }^{[11,12]}$. Obviously, the reactive power characteristics are determined by control mode of DC system. Formulas about inverter-side Q-V relationship under different control modes are derived as below, with subscript $s$ indicating a constant value.

CC-CV Mode. Under this control mode, the inverter reactive power can be formulated as

$Q_{d i}=P_{d i} \tan \varphi_{i}$,

where, $\varphi_{i}$ is the inverter-side power factor angle.

Based on the mathematical model of DC system, the following equation can be derived as

$$
\tan \varphi_{i}=\frac{\sqrt{U_{d 0 i}^{2}-U_{d i}^{2}}}{U_{d i}},
$$

where, $U_{d 0 i}$ is the ideal open-circuit DC voltage of inverter side, which is defined as

$U_{d 0 i}=\frac{3 \sqrt{2}}{\pi} K N U_{L i}$,

where, $K$ is the converter transformer ratio; $N$ is the number of converter bridges; $U_{L i}$ is the AC voltage on inverter bus. In general, $K$ and $N$ is constant, respectively.

Thus, the inverter-side reactive power under CC-CV mode can be formulated as

$Q_{d i}=U_{d s} I_{d s} \frac{\sqrt{U_{d 0 i}^{2}-U_{d s}^{2}}}{U_{d s}}=I_{d s} \sqrt{\left(\frac{3 \sqrt{2}}{\pi} K N U_{L i}\right)^{2}-U_{d s}^{2}}$.

From (4), it's known that the inverter-side reactive power $Q_{d i}$ only depends on inverter bus voltage $U_{L i}$. If $U_{L i}$ drops with the increasing of load, the reactive power consumed by DC system $Q_{d i}$ would decrease. This fact means that $\mathrm{CC}-\mathrm{CV}$ mode can prevent the further drop of AC voltage therefore maintain the voltage stability.

CP-CV Mode. Under this control mode, the rectifier-side active power $P_{d r}$ is a constant value $P_{d s}$. Then the following equation can be derived as

$$
P_{d s}=U_{d r} I_{d}=\left(U_{d s}+I_{d} R_{d c}\right) I_{d},
$$

where, $R_{d c}$ is the resistance of DC line.

As (5) is a quadratic equation, the DC current $I_{d}$ can be solved as

$$
I_{d}=\frac{\sqrt{U_{d s}^{2}+4 P_{d s} R_{d c}}-U_{d s}}{2 R_{d c}} \text {. }
$$

It can be concluded from (6) that $I_{d}$ is also a constant, which can be noted as $I_{d s}^{\prime}$. Based on (4) and (6), the inverter-side reactive power under CP-CV mode can be formulated as

$$
Q_{d i}=I_{d s}^{\prime} \sqrt{\left(\frac{3 \sqrt{2}}{\pi} K N U_{L i}\right)^{2}-U_{d s}^{2}}=\frac{\sqrt{U_{d s}^{2}+4 P_{d s} R_{d c}}-U_{d s}}{2 R_{d c}} \times \sqrt{\left(\frac{3 \sqrt{2}}{\pi} K N U_{L i}\right)^{2}-U_{d s}^{2}} .
$$

Based on (7), we can conclude that the inverter-side reactive power $Q_{d i}$ only depends on inverter bus voltage $U_{L i}$. With the same reason as CC-CV mode, CP-CV mode also has the ability to maintain voltage stability.

CC-CEA Mode. The inverter-side DC voltage can be defined as

$$
U_{d i}=\frac{3 \sqrt{2}}{\pi} K N U_{L i} \cos \gamma_{s}-I_{d s} R_{c},
$$

where, $\gamma_{s}$ is the extinction angel, which is a constant under this control mode; $R_{c}$ is equivalent commutation resistance. 
Based on (3), (4) and (8), the inverter-side reactive power under CC-CEA mode can be derived as $Q_{d i}=I_{d s} \sqrt{\left(\frac{3 \sqrt{2}}{\pi} K N U_{L i}\right)^{2}-\left(\frac{3 \sqrt{2}}{\pi} K N U_{L i} \cos \gamma_{s}-I_{d s} R_{c}\right)^{2}}$.

It's obvious that the inverter-side reactive power $Q_{d i}$ only depends on inverter bus voltage $U_{L i}$. Based on (9) and the characteristic of quadratic equation, when inverter voltage $U_{L i}$ drops, the changes of reactive power depends on the actual operating status. The demand of reactive power may increase in some cases and decrease in other cases. Thus, the influence of CC-CEA mode on voltage stability depends on the system status.

CP-CEA Mode. Under this control mode, the rectifier-side active power $P_{d r}$ is a constant value $P_{d s}$. Then the following equation can be derived as

$$
P_{d s}=\left(U_{d i}+I_{d} R_{d c}\right) I_{d}=\left(\frac{3 \sqrt{2}}{\pi} K N U_{L i} \cos \gamma_{s}-I_{d} R_{c}+I_{d} R_{d c}\right) I_{d} .
$$

Equation (10) can be regarded as a quadratic equation with the variation $I_{d}$. Then $I_{d}$ can be formulated as the function of $U_{L i}$.

$$
I_{d}=\frac{\sqrt{\left(\frac{3 \sqrt{2}}{\pi} K N U_{L i} \cos \gamma_{s}\right)^{2}+4 P_{d s}\left(R_{d c}-R_{c}\right)}-\frac{3 \sqrt{2}}{\pi} K N U_{L i} \cos \gamma_{s}}{2\left(R_{d c}-R_{c}\right)}=f\left(U_{L i}\right) .
$$

Thus, the inverter-side reactive power under CP-CEA mode can be formulated based on (9).

$$
Q_{d i}=f\left(U_{L i}\right) \times \sqrt{\left(\frac{3 \sqrt{2}}{\pi} K N U_{L i}\right)^{2}-\left(\frac{3 \sqrt{2}}{\pi} K N U_{L i} \cos \gamma_{s}-f\left(U_{L i}\right) \times R_{c}\right)^{2}} .
$$

From (12), it's known that the inverter-side reactive power $Q_{d i}$ is the function of inverter bus voltage $U_{L i}$. Based on (11) and (12), it can be concluded when $U_{L i}$ drops, $I_{d}$ would increase, and the reactive power consumed by DC system would increase, which makes $U_{L i}$ drop further. This fact means CP-CEA mode is harmful to the voltage stability of AC system.

\section{MISCR Considering Control Modes of DC System}

Based on the analysis above, reactive power consumed by inverter $Q_{d i}$ only depends on the AC line voltage $U_{L i}$ on inverter bus, no matter what control mode the DC system is under. Therefore, the inverter-side reactive power can be defined as a unary function of $U_{L i}$.

$$
Q_{d i}=f\left(U_{L i}\right) \text {, }
$$

where, the specific form of the function depends on control mode.

The AC/DC hybrid power system must meet the power flow equations when the whole system is in steady state. If the PQ decoupled method is used, the power flow mathematical model is just as follows,

$$
\left[\begin{array}{l}
\Delta P \\
\Delta Q
\end{array}\right]=\left[\begin{array}{ll}
H & 0 \\
0 & L
\end{array}\right]\left[\begin{array}{l}
\Delta \theta \\
\Delta U / U
\end{array}\right] \text {. }
$$

If there is no active power change at all converter AC buses, then it may be assumed that $\Delta P=0$ and (14) reduces to

$$
\Delta Q=L \frac{\Delta U}{U} \text {. }
$$

Equation (15) can be rewritten as

$$
\Delta Q / U=B \Delta U \text {. }
$$

In the power flow mathematical model of AC/DC hybrid system, the node power equation of pure AC nodes are just as usual,

$$
\Delta Q_{i}=Q_{i 0}-U_{i} \sum_{j=1}^{n_{a}} U_{j} B_{i j}=0 .
$$


For the nodes link to converter buses, the DC-consumed reactive power $Q_{d i}$ and the compensation reactive power $\omega C_{i} U_{i}^{2}$ should be taken into consideration. Thus, the node power equation is formulated as

$$
\Delta Q_{i}=Q_{i 0}-U_{i} \sum_{j=1}^{n_{a}} U_{j} B_{i j}-Q_{d i}-\omega C_{i} U_{i}^{2}=Q_{i 0}-U_{i} \sum_{j=1}^{n_{a}} U_{j} B_{i j}-Q_{i d}\left(U_{i}\right)=0 .
$$

Based on (16), (17) and (18), the iteration coefficient of power flow calculation is derived as

$$
B_{i j}^{\prime}=\left\{\begin{array}{l}
B_{i j} \quad(j \neq i) \\
B_{i i}+\frac{1}{U_{i}} \frac{\partial Q_{i d}}{\partial U_{i}} \quad(j=i),
\end{array}\right.
$$

where, the modified item $\frac{1}{U_{i}} \frac{\partial Q_{i d}}{\partial U_{i}}$ depends on the control mode of DC system and the capacity of compensation capacitance.

Considering a small increment in the AC reactive power at $i$-th converter only, the power flow equation can be written as

$$
\left[\begin{array}{l}
0 \\
\cdots \\
d Q_{i} / U_{L i} \\
\cdots \\
0
\end{array}\right]=B^{\prime}\left[\begin{array}{l}
d U_{L 1} \\
\cdots \\
d U_{L i} \\
\cdots \\
d U_{L n}
\end{array}\right] .
$$

If both sides of (20) are multiplied by $1 / d U_{L i}$, the MIIF index of HVDC links respect to the $i$-th HVDC can be obtained as

$$
\left[\begin{array}{l}
M I I F_{1 i} \\
\ldots \\
1 \\
\cdots \\
M I I F_{n 1}
\end{array}\right]=\frac{1}{d U_{L i}}\left(B^{\prime}\right)^{-1}\left[\begin{array}{l}
0 \\
\cdots \\
d Q_{i} / U_{L i} \\
\cdots \\
0
\end{array}\right]=\frac{1}{d U_{L i}} \frac{d Q_{i}}{U_{L i}}\left[\begin{array}{l}
M_{1 i} \\
\cdots \\
M_{k i} \\
\cdots \\
M_{n i}
\end{array}\right],
$$

where, $M_{k i}$ is the element of matrix $M$, which is the inverse matrix of $B^{\prime}$.

The MISCR definition proposed by CIGRE is as below,

$$
\text { MISCR }=\frac{S_{a c i}}{P_{d c i}+\sum_{j=1, j \neq i}^{n} M I I F_{j i} \times P_{d c j}} .
$$

Obviously, the key factor of this definition is MIIF. From (14) to (21), a new analytical equivalent of MIIF is defined. Based on the new MIIF index, a new definition of MISCR can be founded.

$$
\operatorname{MISCR}_{i}=\frac{S_{a c i}}{\frac{d Q_{i}}{d U_{L i}} \frac{1}{U_{L i}} \mathbf{P}_{\mathrm{dc}} \mathbf{M}_{\mathbf{i}}}=\frac{S_{a c i}}{B_{i i}^{\prime} \mathbf{P}_{\mathrm{dc}} \mathbf{M}_{\mathbf{i}}},
$$

where, $\mathbf{P}_{\mathrm{dc}}$ is the row vector of DC active power; $\mathbf{M}_{\mathbf{i}}$ is the $i$-th column of matrix $M$.

Compared to the MISCR with the form of impedance ratio, the new MISCR defined by (23) takes the dynamic characteristics of the inverter-side reactive power into consideration. It gives a comprehensive insight to the interaction between AC and DC systems. After the control modes of all of the HVDC links are determined, the modified iteration coefficient matrix $B^{\prime}$ is a constant matrix, which makes the calculation of the new MISCR easy and clear.

MISCR can reflect the voltage support capacity of AC system, which means the higher the voltage stability is, the greater the value of MISCR is ${ }^{[5]}$. Based on the voltage stability analysis above, we can analyze the characteristics of values of new MISCR of all the four control modes.

(1) The new MISCR is an equivalent of the old one with the form of impedance ratio. Thus, the value of new MISCR must be close to the old one, but there must exist difference depending the control mode. 
(2) CC-CV mode and CP-CV mode can strengthen the voltage stability, which means the new MISCR under these two modes have larger values.

(3) CP-CEA mode would weaken the voltage stability, which results in smaller values of the new MISCR.

(4) The influence of CC-CEA mode on voltage stability depends on the system status. Thus, the value characteristics of new MISCR can’t be determined only according to the control mode.

\section{Case Study}

The power grid of Jiangsu province is used as the example, in which seven HVDC links terminates. The inverter-side control modes of all of these DC links are CEA modes. Considering the reliability and complexity of control system, CEA mode is widely adopted for inverters in practical DC system. Thus we only consider the MISCR characteristics under CC-CEA mode and CP-CEA mode.

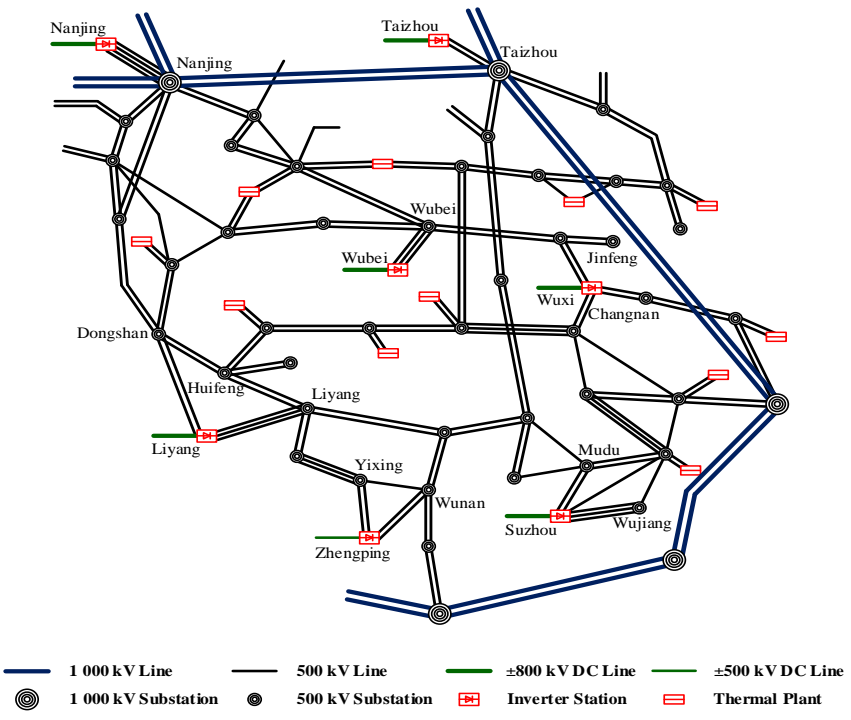

Fig.1 The power grid of Jiangsu province

Based on the formulas derived above, the calculation results are shown in Table 1.

Table 1 MISCR of DC systems terminating in Jiangsu power grid

\begin{tabular}{|c|c|c|c|}
\hline Converter station & Old MISCR & $\begin{array}{c}\text { New MISCR under } \\
\text { CC-CEA mode }\end{array}$ & $\begin{array}{c}\text { New MISCR under } \\
\text { CP-CEA mode }\end{array}$ \\
\hline Nanjing & 4.591 & 4.390 & 3.982 \\
\hline Taizhou & 4.022 & 3.653 & 3.568 \\
\hline Wubei & 3.787 & 3.543 & 3.351 \\
\hline Wuxi & 4.520 & 4.218 & 4.181 \\
\hline Liyang & 3.501 & 3.417 & 3.433 \\
\hline Zhengping & 4.811 & 4.765 & 2.689 \\
\hline Suzhou & 3.024 & 2.957 & 2.896 \\
\hline
\end{tabular}

Based on Table 1, we can draw conclusions below:

(1) Although difference exits, the values of new MISCR of these converter stations is close to old ones, which means the new MISCR can reflect the interaction between AC and DC systems as well as the old ones.

(2) For most converter stations, the new MISCR values of CP-CEA mode are smaller than CC-CEA mode, which validates the analysis that CP-CEA mode is harmful to the voltage stability.

(3) In some converter stations, the new MISCR values of CC-CEA mode are quite close to the ones of CP-CEA mode. But in most station, CC-CEA mode has larger values. This fact validates that the influence of CC-CEA depends on the system status. 


\section{Summary}

By analyzing external reactive power characteristics under four different control modes, the Q-V relationship between inverter-side reactive power and inverter bus voltage is derived. Based on formulas of Q-V relationship and power flow model of PQ decoupled method, the new MISCR is defined after the control mode of each DC system is determined. As MISCR reflects the voltage stability, the characteristics of values of the new MISCR under different modes can be derived. The case study validates the MISCR characteristics for inverters with CEA control mode.

\section{References}

[1] D. Huang, Y. Shu, J. Ruan, and Y. Hu. Ultra high voltage transmission in China: Developments, current status and future prospects. Proceedings of the IEEE, vol. 97, no. 3, pp. 555-583, Mar. 2009.

[2] L. Denis, G. Andersson, Voltage stability analysis of multi-infeed HVDC systems. IEEE Transaction on Power Delivery, vol. 12, no. 1, pp. 547-557, Jan. 1997.

[3] L. Denis, G. Andersson, Power stability analysis of multi-infeed HVDC systems. IEEE Transaction on Power Delivery, vol. 13, no. 1, pp. 923-931, Jan. 1998.

[4] I. T. Fernando, K. L. Kent, J. B. Davies, et al. Parameters for planning and evaluation of multi-infeed HVDC schemes. 2007 Cigre Symposium Osaka, Japan, Nov. 2007.

[5] CIGRE Working Group B4.41. Systems with multiple DC infeed. CIGRE, Paris, France, Tech. Rep. 364, Dec. 2008.

[6] de Toledo P. F., B. Bergdahl, and G. Asplund. Multiple Infeed Short Circuit Ratio-Aspects Related to Multiple HVDC into One AC Network. Transmission and Distribution Conference and Exhibition: Asia and Pacific, 2005 IEEE/PES. 2005.

[7] W. Lin, Y. Tang, G. Bu, et al. Voltage stability analysis of multi-infeed AC/DC power system based on multi-infeed short circuit ratio. Power System Technology (POWERCON), 2010 International Conference on. IEEE, 2010.

[8] D. Yang, K. Zhao, and Y. Liu. Coordinated optimization for controlling short circuit current and multi-infeed DC interaction. Journal of Modern Power Systems and Clean Energy, 2014. 2(4): p. $1-11$.

[9] Y. Shao, Y. Tang. Voltage stability analysis of multi-infeed HVDC systems using small-signal stability assessment. Transmission and Distribution Conference and Exposition, 2010 IEEE PES. IEEE, 2010: 1-6.

[10]Y. Jun, M. Shicong, and D. Mian. Study on operation characteristics and stability evaluation method at sending-end of DC transmission system. Power System Technology (POWERCON), 2014 International Conference on. IEEE, 2014: 696-699.

[11]H. P. LIPS. Aspects of multiple infeed of HVDC inverter station into a common AC system. IEEE Transactions on Power Apparatus, 1973, 92(2):135-141.

[12]M. Szechtman, L. A. S. Pilotto, and W. W. Ping. The behavior of several HVDC links terminating in the same load area. Paris: CIGRE, 1992, Group 14. 